Publ. RIMS, Kyoto Univ.

17 (1981), 201-211

\title{
Eta Invariants and Conformal Immersions
}

By

\author{
Kenji TsuboI*
}

\section{§o. Introduction}

Let $M$ be a closed oriented Riemannian manifold of dimension $4 k-1$. The purpose of this paper is to give necessary conditions of the existence of a global conformal immersion of $M$ in an appropriate Euclidean space in terms of the eta invariants and to give examples by applying these results. Our main results are Theorem 3.10 and Theorem 3.12.

In their paper [1], Atiyah, Patodi and Singer defined a real ralued spectral invariant of $M I$ which is called the eta invariant of $M$. Throughout this paper $\eta(M)$ denotes the eta invariant of $M . \eta(M)$ can be calculated in some cases. Let $\widetilde{M}$ be a regular covering space over $M$ with finite covering group. We assume that the orientations and the Riemannian metrics of $\widetilde{M}$ and $M$ are compatible by the covering projection. Moreover we assume that $\widetilde{M}$ admits an orientation-reversing isometry. Then, the formula in [7] enable us to calculate $\eta(M)$ with respect to any Riemannian metric of $M$. In Section 1, we recall the notion of the eta invariant and give an example of the calculation.

On the one hand, in his paper [11], Simons defined a singular $\boldsymbol{R} / \boldsymbol{Z}$-cochain on $M$ which is called the $S$-character or the Chern-Simons invariant. The $S$-character is an obstruction to the existence of a global conformal immersion of $M$ in an appropriate Euclidean space. In many cases, however, it is difficult to calculate the $S$-characters and they seem to be not calculated with respect to non-standard metrics. In Section 2, we recall the notion of the $S$-character.

In Section 3, by connecting the $S$-character and the eta invariant, we give necessary conditions of the existence of a global conformal

Communicuted by N. Shimada, January 28, 1980.

* Department of Mathematics, Kyoto University, Kyoto 606, Japan. 
immersion of $M$ in an appropriate Euclidean space in terms of the eta invariants.

In Section 4, we give examples by applying the results in Section 3.

I would like to thank Professor Adachi who gave me the opportunity of doing this work and Dr. Kôno who gave me many valuable advices.

\section{$\S 1$. The Eta Invariants}

Let $M$ be a closed oriented Riemannian manifold of dimension $4 k-1$ and $\Lambda^{e v}(M)$ the exterior algebra which consists of differential forms on $M$ having type $2 p$ for some $0 \leqq p \leqq 2 k-1$. Let $D$ denote the first order self-adjoint elliptic differential operator on $\Lambda^{e v}(M)$ given by

$$
\phi \mapsto(-1)^{k+p+1}(* d-d *) \phi
$$

where $\phi$ is a $2 p$-form and $*$ is Hodge's star operator. Then $D$ has the pure point spectrum consisting of eigenvalues $\lambda$ with finite multiplicity $m(\lambda)$. The spectral function

$$
\eta(s, M)=\sum_{\lambda \neq 0}(\lambda /|\lambda|) m(\lambda)|\lambda|^{-s}
$$

converges for $\operatorname{Re}(s)$ sufficiently large and has a meromorphic continuation to the entire complex $s$-plane. Moreover, 0 is not a pole and $\eta(0, M)$, which we call the eta invariant of $M$ and denote by $\eta(M)$, is finite. The main result of [1] is as follows.

Theorem 1. 1 (Atiyah-Patodi-Singer). Let (W, $\widehat{g})$ be a compact oriented 4k-dimensional Riemannian manifold with boundary $M$ and assume that, near $M$, it is isometric to a product. Then

$$
\eta(M)=\int_{W} L_{k}\left(P_{1}(\widehat{g}), \cdots, P_{k}(\widehat{g})\right)-\operatorname{sign}(W)
$$

where $\operatorname{sign}(W)$ is the signature of $W, L_{k}$ is the $k$-th Hirzebruch L-polynomial and $P_{i}(\widehat{g})$ is the $i$-th Pontrjagin form.

Remark 1.2. Even if $M$ is not zero-cobordant, $2 M=M \cup M$ is zero-cobordant by the dimensional reason. Then, let $2 M=\partial W$, as it is 
obvious from the definition that $\eta(2 M)=2 \eta(M)$, we have

$$
\eta(M)=\frac{1}{2} \eta(2 M)=\frac{1}{2}\left\{\int_{W^{*}} L_{k}\left(P_{1}(\widehat{g}), \cdots, P_{k}(\widehat{g})\right)-\operatorname{sign}(W)\right\} .
$$

Remark 1.3. It can be proved using Theorem 1.1 and the conformal invariance of Pontrjagin forms that $\eta(M)$ is a conformal invariant. Namely, if two Riemannian metrics $g, g^{\prime}$ of $M$ are conformally related (i.e. there exists a positive real valued smooth function $f$ on $M$ such that $\left.g^{\prime}=f \cdot g\right)$, then $\eta\left(M_{g}\right)=\eta\left(M_{g^{\prime}}\right)$.

Now we suppose that $\widetilde{M}$ is a closed oriented Riemannian manifold of dimension $4 k-1$ and $G$ is a finite group which acts on $\widetilde{M}$ freely, isometrically and orientation-preservingly. $\widetilde{M}$ or $2 \widetilde{M}$ is zero-cobordant by the dimensional reason. For simplicity, we assume that $\widetilde{M}$ is zerocobordant. Let $\widetilde{W}$ be a compact oriented $4 k$-dimensional Riemannian manifold with boundary $\widetilde{M}$. We assume that the action of $G$ is extended to an isometric action on $\widetilde{W}$.

Let $G \ni h \neq 1$. Then the fixed point set $F$ of $h$ is the disjoint union of closed connected totally geodesic (but not necessarily orientable) submanifolds $N$ of $\widetilde{W}$. Let $[N]$ denote the (twisted) fundamental homology class of $N$. The normal bundle $T N^{\perp}$ of $N$ decomposes as

$$
T N^{\perp}=T N^{\perp}(-1) \oplus\left(\oplus_{i} T N^{\perp}\left(\omega_{i}\right)\right)
$$

where the differential of $h$ acts on $T N^{\perp}(-1)$ via multiplication by -1 and on $T N^{\perp}\left(\omega_{i}\right)$ via rotation through the constant angle $\omega_{i}, \omega_{i} \neq \pi$. The complex vector bundle $T N^{\perp}\left(\omega_{i}\right)$ has the natural orientation and we assume that the (local) orientations of $T N^{\perp}(-1)$ and $[N]$ are compatible with the orientation of $\widetilde{W}$.

Now, corresponding to the $G$-signature theorem of Atiyah-Singer in their paper [3], let $\eta(h, \widetilde{M})$ denote the difference

$$
\begin{aligned}
& \sum_{N \in F}\left\{2^{(n-m) / 2} \prod_{i}\left(-\sqrt{-1} \cot \left(\omega_{i} / 2\right)\right)^{c\left(\omega_{i}\right)} \mathcal{L}(T N) \mathcal{L}\left(T N^{\perp}(-1)\right)\right. \\
& \left.\times e\left(T N^{\perp}(-1)\right) \prod_{i} \mathscr{M}^{\omega_{i}}\left(T N^{\perp}\left(\omega_{i}\right)\right)\right\}[N]-\operatorname{sign}(h, \widetilde{W})
\end{aligned}
$$

where $n$ is the dimension of $N, m$ is the fiber dimension of $T N^{\perp}(-1)$, $c\left(\omega_{i}\right)$ is the complex fiber dimension of $T N^{\perp}\left(\omega_{i}\right), e$ is the (twisted) 
Euler class, $\mathcal{L}$ and $\mathscr{M}^{\omega_{i}}$ are the stable characteristic classes defined in $[3, \S 6]$ (or $[7, \S 2]$ ) and $\operatorname{sign}(h, \widetilde{W})$ is the $G$-signature of $\widetilde{W}$ on $h \in G$.

Then, in his paper [7], Donnelly gave the following formula which is very useful.

$$
\eta(\widetilde{M} / G)=(1 /|G|)\left\{\eta(\widetilde{M})+\sum_{h \neq 1} \eta(h, \widetilde{M})\right\} .
$$

where $|G|$ is the order of $G$. In particular, if $\widetilde{M}$ admits an orientationreversing isometry, it is obvious from the definition of $\eta(\widetilde{M})$ that $\eta(\widetilde{M})$ is zero. Then

$$
\eta(\widetilde{M} / G)=(1 /|G|) \sum_{h \neq 1} \eta(h, \widetilde{M}) .
$$

Details may be found in [7].

Example 1. 7. Let

$$
S^{4 k-1}=\left\{\left(z_{1}, \cdots, z_{2 k}\right) \in C^{2 k} ;\left|z_{1}\right|^{2}+\cdots+\left|z_{2 k}\right|^{2}=1\right\}
$$

be the unit sphere in $C^{2 k}$. Suppose that $p$ is a positive integer and $1 \leqq q_{1}, \cdots, q_{2 k} \leqq p-1$ are integers which are relatively prime to $p$. The cyclic group $G=\boldsymbol{Z} / p \boldsymbol{Z}$ acts on $S^{4 k-1}$ by sending a generator of $G$ to the diffeomorphism

$$
\left(\approx_{1}, \cdots, z_{2 k}\right) \mapsto\left(\left(\exp \left(2 \pi \sqrt{-1} q_{1} / p\right)\right) z_{1}, \cdots,\left(\exp \left(2 \pi \sqrt{-1} q_{2 k} / p\right)\right) z_{2 k}\right)
$$

for $\left(z_{1}, \cdots, z_{2 k}\right) \in S^{4 k-1}$. The quotient space $S^{4 k-1} / G$ will be denoted by $L\left(p ; q_{1}, \cdots, q_{2 k}\right)$. Let $g$ be any Riemannian metric of $L\left(p ; q_{1}, \cdots, q_{2 k}\right)$ which has the following property;

(1. 8) $\left(S^{4 k-1}, r^{*} g\right)$ admits an orientation-reversing isometry where $r: S^{4 k-1}$ $\rightarrow L\left(p ; q_{1}, \cdots, q_{2 k}\right)$ is the covering projection.

$g$ need not be the standard metric of lens spaces. Let

$$
D^{4 k}=\left\{\left(z_{1}, \cdots, z_{2 k}\right) \in C^{2 k} ;\left|z_{1}\right|^{2}+\cdots+\left|z_{2 k}\right|^{2} \leqq 1\right\}
$$

be the unit ball in $C^{2 k}$. Then the action of $G$ on $S^{4 k-1}$ can be extended to the action on $D^{4 k}$ and the fixed point set of $G \ni h \neq 1$ is the origin of $\boldsymbol{C}^{2 k}$.

Connecting the standard metric near the origin with $r^{*} g$ on the boundary $S^{4 k-1}$ and averaging over $G$, we obtain the Riemannian metric 
$g_{D}$ on $D^{4 k}$ which has the following properties;

(1) $g_{D}$ is invariant by the action of $G$,

(2) $g_{D}$ coincides with the standard metric of $\mathbb{C}^{2 k}$ near the origin,

(3) $g_{D}$ coincides with $r^{*} g$ on the boundary $S^{4 k^{-1}}$.

Then according to the formula (1.6), we can calculate the eta invariant.

$$
\eta\left(L\left(p ; q_{1}, \cdots, q_{2 k}\right)\right)=\left((-1)^{k} / p\right) \sum_{s=1}^{p-1} \prod_{j=1}^{2 k} \cot \left(s q_{j} \pi / p\right) .
$$

Remark 1.10. As in Example 1.7, by connecting a metric on $G$ invariant tubular neighborhoods of fixed point manifolds with a metric on the boundary $\widetilde{M}$, it can be proved that, for $G \ni h \neq 1, \eta(h, \widetilde{M})$ does not depend on the $G$-invariant metric of $\widetilde{W}$, namely, $\eta(h, \widetilde{M})$ is a topological invariant of $\widetilde{M}$. This is the fact known in [2], [7] and this fact gives the possibility of calculating eta invariants with respect to nonstandard metrics.

\section{§ 2. The T-Forms and the S-Characters}

Let $\pi: O(M) \rightarrow M$ be the orthonormal frame bundle with fiber $O(4 k-1)$. Let $\theta$ denote the Riemannian connection form in $O(M)$ and $\Omega$ the curvature form of $\theta$. Let $I(m)$ denote the set of invariant polynomials of degree $m$ on the Lie algebra of $O(4 k-1)$. For any $P \in I(m)$, let $P(\Omega)$ denote the $2 m$-form on $M$ defined by the Weil homomorphism. Then it is well known that there exists a $(2 m-1)$-form $T P(\theta)$ on $O(M)$ which has the following properties;

$$
d T P(\theta)=\pi^{*} P(\Omega) .
$$

In particular, $T P(\theta)$ defines a cohomology class

$$
\{T P(\theta)\} \in H^{2 m-1}(O(M) ; \boldsymbol{R}) \quad \text { if and only if } P(\Omega)=0 .
$$

(2.2) Let $P^{\prime} \in I\left(m^{\prime}\right)$. Then

$T\left(P P^{\prime}\right)(\theta)=T P(\theta) \wedge P^{\prime}(\Omega)+$ exact form $=P(\Omega) \wedge T P^{\prime}(\theta)+$ exact form .

Details may be found in $[5, \S 3]$.

Now, let $G(4 k-1, N)$ be a Grassmann manifold for sufficiently 
large $N$ which is a finite approximation to $B O(4 k-1)$ and let $\Omega_{0}$ be the curvature form of the universal connection in the canonical $O(4 k-1)$ bundle over $G(4 k-1, N)$. Let $Z_{2 m-1}(M)$ denote the additive group of the smooth singular $(2 m-1)$-cycles in $M$. Let $u$ be an element of $H^{2 m}(B O(4 k-1) ; \boldsymbol{Z})$ which we can naturally regard as an element of $H^{2 m}(G(4 k-1, N) ; \boldsymbol{Z})$ and $P_{u}$ be the element of $I(m)$ which corresponds to $u$ except 2 -torsion by the Weil homomorphism. We define a $\boldsymbol{Z}$-module homomorphism $s u(\theta): Z_{2 m-1}(M) \rightarrow \boldsymbol{R} / \boldsymbol{Z}$ as follows. Let $\psi(\theta): M \rightarrow G(4 k-1$, $N)$ be the classifying map of $O(M)$ with the Riemannian connection. Namely, the Riemannian connection of $M$ is the pull back connection from the universal connection of $G(4 k-1, N)$. For any $x \in Z_{2 m-1}(M)$, $\psi(\theta)_{*}(x)$ is a $(2 m-1)$-cycle in $G(4 k-1, N)$ and either $\psi(\theta)_{*}(x)$ or $2 \psi(\theta)_{*}(x)$ bounds. If $\psi(\theta)_{*}(x)$ bounds, say $\psi(\theta)_{*}(x)=\partial y$ where $y$ is a smooth singular $2 m$-chain, define

$$
s u(\theta)(x)=\overline{\int_{y} P_{u}\left(\Omega_{0}\right)}
$$

where - denotes reduction $\bmod \boldsymbol{Z}$. If $2 \psi(\theta)_{*}(x)$ bounds, we choose an integral cochain $U$ which represents $u$ and define

$$
s u(\theta)(x)=\overline{\frac{1}{2}\left\{\int_{y} P_{u}\left(\Omega_{0}\right)-U(y)\right\}} .
$$

This definition of $\operatorname{su}(\theta)$ is independent of the choices of $\psi(\theta), y$ and $U$. Details may be found in $[11, \S 3]$.

As $\boldsymbol{R} / \boldsymbol{Z}$ is a divisible $\boldsymbol{Z}$-module $s u(\theta)$ can be extended to an $\boldsymbol{R} / \boldsymbol{Z}$ cochain with the ambiguity of a coboundary. $s u(\theta)$ has the following properties;

$$
\delta(\operatorname{su}(\theta))=\overline{P_{u}(\Omega)} .
$$

In particular, $s u(\theta)$ defines an element of $H^{2 m-1}(M ; \boldsymbol{R} / \boldsymbol{Z})$ if and only if $P_{u}(\Omega)=0$.

(2.4) If $P_{u}(\Omega)=0$, the lift of the cohomology class $\{s u(\theta)\}$ to $O(M)$ coincides with the reduction $\bmod Z$ of $\left\{T P_{u}(\theta)\right\}$.

Now let $Q\left(a_{1}, \cdots, a_{k}\right)$ be any integral polynomial (i.e. polynomial 
over the integers) of weight $k$ (i.e. $Q\left(a_{1}^{4}, \cdots, a_{k}^{4 k}\right)$ is homogeneous of degree $4 k)$. Let $H^{4 i}(B O(4 k-1) ; Z \mathbb{Z}) \ni p_{i}^{+}$be the $i$-th inverse Pontrjagin class defined by

$$
p_{i}^{\perp}=(-1)^{i} B t^{*}\left(c_{2 i}^{\frac{1}{}}\right)
$$

where $t: O(4 k-1) \rightarrow U(4 k-1)$ is the natural inclusion and $c_{2 i}^{\frac{1}{1}} \in$ $H^{4 i}(B U(4 k-1) ; Z ⿱ Z)$ is the $2 i$-th inverse Chern class characterized by $\left(1+c_{1}+\cdots+c_{j}+\cdots\right)\left(1+c_{1}^{\perp}+\cdots+c_{j}^{\perp}+\cdots\right)=1$ in integral domain $H^{*}(B U(4 k-1) ; Z \mathbb{Z})$. Then $Q\left(p_{1}^{\perp}, \cdots, p_{\frac{1}{k}}\right)$ is an element of

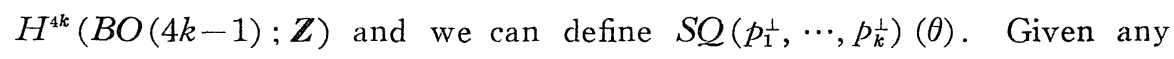
$1 \leqq s \leqq k$, evaluating on the top cycle $M$, we define $S Q\left(p_{s}^{\perp}, \cdots, p_{k}^{\perp}\right)(M)$ which is an element of $\boldsymbol{R} / \boldsymbol{Z}$.

The key theorem of this paper is the following which is the result in [11, Theorem 5.4].

Theorem 2.6 (Simons). A necessary condition that $M$ admits a global conformal immersion with codimension $d$ in $\boldsymbol{R}^{4 k-1+d}$ is that $S Q\left(p_{s}^{\perp}, \cdots, p_{k}^{\perp}\right)(M)=0$ for any $s \geqq[d / 2]+1$ and any $Q$ as above.

\section{$\S$ 3. The Eta Invariants and the Conformal Immersions}

From the result in [11, Theorem 5.15], the following lemma follows immediately.

Lemma 3. 1. We assume that $M$ is the boundary of $W$ and that, near $M,(W, \widehat{g})$ is isometric to a product. Then, for any $u \in H^{4 k}(B O(4 k-1) ; Z)$,

$$
\boldsymbol{R} / \boldsymbol{Z} \ni s u(M)=\overline{\int_{W} P_{u}(\widehat{g})}
$$

where, as in Theorem 1.1, $P_{u}(\widehat{g})$ denotes the $4 k$-form defined by the Weil homomorphism with respect to the Riemannian metric $\hat{g}$ of $W$.

Remark 3.2. We assume that $M$ is zero-cobordant and $u \in$ $H^{4 k}(B O(4 k-1) ; Z Z)$ is a torsion element. Then $P_{u}=0$ and it follows from Lemma 3.1 that $s u(M)=0$. 
Now, let $N_{k}$ denote the denominator of the $k$-th Hirzebruch $L$ polynomial $L_{k}$. Namely, $N_{k}$ is the least integer for which $N_{k} L_{k}$ is a polynomial over the integers. It is well known that

$$
N_{k}=\prod_{q} q^{[2 k /(q-1)]}
$$

where the product is over odd primes $q, 3 \leqq q \leqq 2 k+1$. For example, $N_{1}=3, N_{2}=45$ etc.

Lemma 3. 3. There exists an integral polynomial $Q_{k}$ of weight $k$ such that $N_{k} L_{k}\left(P_{1}, \cdots, P_{k}\right)=Q_{k}\left(P_{1}^{\perp}, \cdots, P_{\bar{k}}^{\perp}\right)$ as an element of $I(2 k)$, where $P_{i} \in I(2 i)$ is the $i$-th Pontrjagin polynomial and $P_{i}^{\perp} \in I(2 i)$ is the $i$-th inverse Pontrjagin polynomial.

Proof. From the relation $\left(1+P_{1}+\cdots+P_{i}+\cdots\right)\left(1+P_{1}^{\perp}+\cdots+P_{i}^{\perp}\right.$ $+\cdots)=1$, it follows that $P_{i}=-P_{i}^{\perp}-P_{i-1}^{\perp} P_{1}-\cdots-P_{1}^{\perp} P_{i-1}$. Hence, it follows by the induction that $P_{i}$ can be expressed as the integral polynomial of $P_{i}^{i}, \cdots, P_{i}^{\perp}$. Therefore, the lemma follows immediately.

Lemma 3. 4. $Q_{k}\left(P_{1}^{\perp}, \cdots, P_{\frac{1}{k}}^{\perp}\right)=n_{k} P_{k}^{\perp}+$ decomposable part, for a certain integer $n_{k}$. The decomposable part is the Z-linear combination of terms any of which can be decomposed as the product

$$
R\left(P_{1}, \cdots\right) R^{\prime}\left(P_{1}, \cdots, P_{[k / 2]}\right),
$$

where $R$ is an integral monomial of weight $\geqq[k+1 / 2]$ and $R^{\prime}$ is an integral monomial of weight $\leqq[k / 2]$.

Proof. As in Lemma 3.3, $P_{\bar{i}}^{+}$can be expressed as the integral polynomial of $P_{1}, \cdots, P_{i}$ for $1 \leqq i \leqq k-1$. Hence, $Q_{k}\left(P_{1}^{\perp}, \cdots, P_{k}^{\perp}\right)=$ $n_{k} P_{k}^{\perp}+Z$-linear combination of $P_{1} P_{k-1}, \cdots, P_{[k+1 / 2]} P_{[k / 2]}, \cdots,\left(P_{1}\right)^{k}$.

Remark 3. 6. In $H^{4 k}(B O(4 k-1) ; \boldsymbol{Z})$, the equality

$$
\begin{aligned}
N_{k} L_{k}\left(p_{1}, \cdots, p_{k}\right) & =Q_{k}\left(p_{1}^{\perp}, \cdots, p_{k}^{\perp}\right)=n_{k} p_{k}^{\perp} \\
& + \text { decomposable part of type }(3.5)
\end{aligned}
$$

still holds modulo 2-torsion elements. Hence, 


$$
\begin{aligned}
2 N_{k} L_{k}\left(p_{1}, \cdots, p_{k}\right) & =2 Q_{k}\left(p_{1}^{\perp}, \cdots, p_{\frac{\perp}{k}}^{\perp}=2 n_{k} p_{k}^{\perp}\right. \\
& + \text { decomposable part of type (3.5). }
\end{aligned}
$$

It is well known that the Pontrjagin forms of Riemannian metrics are conformally invariant and if $M$ is conformally flat, namely if $M$ is locally conformally isomorphic to the flat Euclidean space, then $P_{i}(\Omega)=0$ for any $i \geqq 1$.

Defimition 3.7. In this paper, we will call a $(4 k-1)$-dimensional Riemannian manifold $M$ to be "partially Pontrjagin flat" if the Riemannian metric of $M$ satisfies the following condition;

$$
R\left(P_{1}(\Omega), \cdots, P_{j}(\Omega)\right)=0
$$

as differential $4 j$-form on $M$ for any integral monomial $R$ of weight j. $[k+1,2] \leqq j \leqq k-1$.

Example 3.9. If $k \geqq 3$, decomposing $\mathbb{R}^{4 k-1}=\mathbb{R}^{4 k-5} \times \mathbb{R}^{4}$ where we suppose that $\mathbb{R}^{4 k-5}$ has the standard flat metric and $\mathbb{R}^{4}$ has a metric with respect to which the first Pontrjagin form does not vanish, it can be proved that $\mathbb{R}^{ \pm k-1}$ has a metric which is partially Pontrjagin flat, but not conformally flat. Thus, using this and destroying the standard metrics locally, we can construct Riemannian metrics of lens spaces which are partially Pontrjagin flat, but not conformally flat, and have the property (1. 8).

Theorem 3. 10. We assume thai $M$ is partially Pontrjagin flat and $\pi^{*}: H^{4 k-1}(M ; \mathbb{R} / \mathbb{Z}) \rightarrow H^{4 k-1}(O(M) ; \mathbb{R} / \mathbb{Z})$ is injective, where $\pi: O(M) \rightarrow M$ is the orthonormal frame bundle. Then, a necessary condition that $M$ admits a global conformal immersion with codimension $2 k-1$ in $\mathbb{R}^{6 k-2}$ is that $N_{k} \eta(M)$ is an integer if $M$ is zerocobordant and $2 N_{k}^{\top} \eta(M)$ is an integer if $M$ is not zero-cobordant.

Proof. If $R\left(P_{1}(\Omega), \cdots\right)$ vanishes, according to the product formula (2.2) of $T$-forms, we have

$$
\left\{T\left(R\left(P_{1}, \cdots\right) R^{\prime}\left(P_{1}, \cdots\right)\right)(\theta)\right\}=\left\{R\left(P_{1}(\Omega), \cdots\right) \wedge T R^{\prime}\left(P_{1}, \cdots\right)(0)\right\}=0 .
$$


Hence, considering the decomposition (3.5) of the decomposable part of $Q_{k}\left(P_{1}^{\perp}, \cdots, P_{k}^{\perp}\right)$, it follows from the assumption that the cohomology class $\left\{T Q_{k}\left(P_{1}^{\perp}, \cdots, P_{k}^{\perp}\right)(\theta)\right\}$ coincides with $n_{k}\left\{T P_{\frac{\perp}{k}}^{\perp}(\theta)\right\}$. Therefore, it follows from (2.4) that

$$
\begin{aligned}
& \pi^{*}\left\{S N_{k} L_{k}\left(p_{1}, \cdots, p_{k}\right)(\theta)\right\}=\pi^{*}\left\{S Q_{k}\left(p_{1}^{\perp}, \cdots, p_{k}^{\perp}\right)(\theta)\right\} \\
& =\overline{\left\{T Q_{k}\left(P_{1}^{\perp}, \cdots, P_{\frac{1}{k}}^{\perp}\right)(\theta)\right\}}=\overline{n_{k}\left\{T P_{\frac{1}{k}}^{\perp}(\theta)\right\}}=\pi^{*}\left(n_{k}\left\{S p_{\frac{1}{k}}^{\perp}(\theta)\right\}\right) .
\end{aligned}
$$

Hence, it follows from the injectivity of $\pi^{*}$ that

$$
\left\{S N_{k} L_{k}\left(p_{1}, \cdots, p_{k}\right)(\theta)\right\}=n_{k}\left\{S p_{k}^{\perp}(\theta)\right\} .
$$

Thus, if $M$ is conformally immersible with codimension $2 k-1$, according to Theorem 2.6, we have

$$
S N_{k} L_{k}\left(p_{1}, \cdots, p_{k}\right)(M)=n_{k} S p_{k}^{\perp}(M)=0 .
$$

We first assume that $M$ is zero-cobordant and $M=\partial W$. From Theorem 1. 1 and Lemma 3.1, it follows that

$$
\begin{aligned}
0 & =S N_{k} L_{k}\left(p_{1}, \cdots, p_{k}\right)(M)=\overline{N_{k} \int_{W} L_{k}\left(P_{1}(\widehat{g}), \cdots, P_{k}(\widehat{g})\right)} \\
& =\overline{N_{k}\left\{\int_{W} L_{k}\left(P_{1}(\widehat{g}), \cdots, P_{k}(\widehat{g})\right)-\operatorname{sign}(W)\right\}}=\overline{N_{k} \eta(M)} .
\end{aligned}
$$

Secondly, if $M$ is not zero-cobordant, $2 M$ is zero-cobordant by the dimensional reason and let $2 M=\partial W$. Then we have

$$
\begin{aligned}
& 0=2 S N_{k} L_{k}\left(p_{1}, \cdots, p_{k}\right)(M)=S N_{k} L_{k}\left(p_{1}, \cdots, p_{k}\right)(2 M) \\
& =\overline{N_{k} \int_{W} L_{k}\left(P_{1}(\widehat{g}), \cdots, P_{k}(\widehat{g})\right)} \\
& =\overline{2 N_{k} \frac{1}{2}\left\{\int_{W} L_{k}\left(P_{1}(\widehat{g}), \cdots, P_{k}(\widehat{g})\right)-\operatorname{sign}(W)\right\}}=\overline{2 N_{k} \eta(M)} .
\end{aligned}
$$

In particular, we consider the case that $k=1$. It is well known that all closed orientable 3-dimensional manifolds are zero-cobordant and parallelizable, hence, satisfy the assumption of Theorem 3.10. As $N_{1}$ $=3$, we obtain the following.

Corollary 3.11. A necessary condition that a closed oriented 3-dimensional manifold admits a global conformal immersion in $\boldsymbol{R}^{4}$ 
is that $3 \eta(M)$ is an integer.

On the one hand, it is well known that all closed orientable 3-dimensional manifolds are smoothly immersible in $\mathbb{R}^{4}$. In Section 4 , as an example of Corollary 3.11, we will examine the conformal immersion of the quotient spaces of $S^{3}$ by binary polyhedral groups.

Theorem 3. 12. We assume that $M$ is partially Pontrjagin flat and there exists an integer $c$ such that $c p_{i}(T M) \in H^{4 i}(M ; \mathbb{Z})$ vanishes for any $1 \leqq i \leqq[k / 2]$ where $p_{i}(T M)$ is the $i$-th Pontrjagin class of the tangent bundle TM. Then, a necessary condition that $M$ admits a global conformal immersion with codimension $2 k-1$ in $\mathbb{R}^{6 k-2}$ is that $c N_{k} \eta(M)$ is an integer if $c$ is even or $M$ is zero-cobordant and $2 c N_{k} \eta(M)$ is an integer if $c$ is odd and $M$ is not zero-cobordant.

Proof. We consider the decomposition (3.5). As $R\left(P_{1}(\Omega), \cdots\right)$ vanishes by the assumption, according to the product formula of the $S$ characters in [4], we have

$$
\begin{aligned}
c\{ & \left.S\left(R\left(p_{1}, \cdots\right) R^{\prime}\left(p_{1}, \cdots, p_{[k / 2]}\right)\right)(\theta)\right\} \\
& =\left\{S R\left(p_{1}, \cdots\right)(\theta)\right\} \cup c R^{\prime}\left(p_{1}(T M), \cdots, p_{[k / 2]}(T M)\right)=0 .
\end{aligned}
$$

We first assume that $c$ is even and $c=2 a$. Then, it follows from Remark 3. 6 that $c\left\{S Q_{k}\left(p_{1}^{\perp}, \cdots, p_{k}^{\perp}\right)(\theta)\right\}=c n_{k}\left\{S p_{k}^{\perp}(\theta)\right\}$ and

$$
c S N_{k} L_{k}\left(p_{1}, \cdots, p_{k}\right)(M)=c S Q_{k}\left(p_{1}^{\perp}, \cdots, p_{k}^{\perp}\right)(M)=c n_{k} S p_{k}^{\perp}(M) .
$$

Thus if $M$ is conformally immersible with codimension $2 k-1$, then $S p_{\frac{1}{k}}(M)=0$ and $c S N_{k} L_{k}\left(p_{1}, \cdots, p_{k}\right)(M)=0$. On the one hand, $2 M$ is zero-cobordant by the dimensional reason. So let $2 M=\partial W$. Then we have

$$
\begin{aligned}
0 & =c S N_{k} L_{k}\left(p_{1}, \cdots, p_{k}\right)(M)=a S N_{k} L_{k}\left(p_{1}, \cdots, p_{k}\right)(2 M) \\
& =\overline{a N_{k} \int_{W} L_{k}\left(P_{1}(\widehat{g}), \cdots, P_{k}(\widehat{g})\right)} \\
& =\overline{c N_{k} \frac{1}{2}\left\{\int_{W} L_{k}\left(P_{1}(\widehat{g}), \cdots, P_{k}(\widehat{g})\right)-\operatorname{sign}(W)\right\}}=\overline{c N_{k} \eta(M)} .
\end{aligned}
$$


Secondly, we assume that $M$ is zero-cobordant and $M=\partial W$. Then it also follows from Remark 3.2 that

$$
c S N_{k} L_{k}\left(p_{1}, \cdots, p_{k}\right)(M)=c n_{k} S p_{\frac{1}{k}}(M) .
$$

Hence, we have

$$
\begin{aligned}
& 0=c S N_{k} L_{k}\left(p_{1}, \cdots, p_{k}\right)(M)=\overline{c N_{k} \int_{W} L_{k}\left(P_{1}(\widehat{g}), \cdots, P_{k}(\widehat{g})\right)} \\
& =\overline{c N_{k}\left\{\int_{W} L_{k}\left(P_{1}(\widehat{g}), \cdots, P_{k}(\widehat{g})\right)-\operatorname{sign}(W)\right\}}=\overline{c N_{k} \eta(M)} .
\end{aligned}
$$

If $c$ is odd and $M$ is not zero-cobordant, we have

$$
2 c S N_{k} L_{k}\left(p_{1}, \cdots, p_{k}\right)(M)=2 c S Q_{k}\left(p_{1}^{\frac{1}{1}}, \cdots, p_{k}^{\perp}\right)(M)=2 c n_{k} S p_{k}^{\perp}(M) .
$$

Hence, we have

$$
\begin{aligned}
0 & =2 c S N_{k} L_{k}\left(p_{1}, \cdots, p_{k}\right)(M)=c S N_{k} L_{k}\left(p_{1}, \cdots, p_{k}\right)(2 M) \\
& =c N_{k} \int_{W} L_{k}\left(P_{1}(\widehat{g}), \cdots, P_{k}(\widehat{g})\right) \\
& =\overline{2 c N_{k} \frac{1}{2}\left\{\int_{W} L_{k}\left(P_{1}(\widehat{g}), \cdots, P_{k}(\widehat{g})\right)-\operatorname{sign}(W)\right\}}=\overline{2 c N_{k} \eta(M)} .
\end{aligned}
$$

Q.E.D.

In Section 4, as an example of this theorem, we will examine the conformal immersion of a certain lens space.

\section{§4. Examples}

As an example of Corollary 3.11, we consider 3-dimensional manifolds of positive constant curvature. They are known to be the quotient spaces of $S^{3}$ by free orthogonal actions of certain finite groups which are completely classified. In his paper [9], Millson calculated the $S$-characters of lens spaces, namely the quotient spaces by cyclic groups, which have the standard Riemannian metrics of positive constant curvature. And he examined their conformal immersions by using those results. So we consider the quotient spaces of $S^{3}$ by binary polyhedral groups.

We regard $S^{3}$ as the Lie group of all quaternions of length one. 
Let $G$ be a finite subgroup of $S^{3}$ which acts on $S^{3}$ on the right. The action of $G$ on $S^{3}$ can be extended to the action on the 4-ball $D^{1}$ and the fixed point set of $G \supseteq h \neq 1$ is the origin of $\mathbb{R}^{4}$. Let $\exp (\sqrt{-1}(0)(h))$ and $\exp (-\sqrt{-1} \omega(h))$ be the eigenvalues of $h \in G$ which can be naturally regarded as an element of $S U(2)$. Then the rotation angles of the action of $h$ around the origin are just $(1)(h)$ and $-\omega(h)$. Hence, according to the formula (1.6), we can calculate the eta invariant of $S^{3} / G$.

$$
\begin{aligned}
& \eta\left(S^{3} / G\right) \\
= & (1 /|G|) \sum_{h \neq 1}\{-\sqrt{-1} \cot (\omega(h) / 2)\}\{-\sqrt{-1} \cot (-\omega(h) / 2)\} \\
= & (1 /|G|) \sum_{h=\neq 1} \cot ^{2}(\omega(h) / 2) .
\end{aligned}
$$

Now, let $O^{*}$ denote the finite subgroup of $S^{3}$ generated by $P=i$, $Q=j, B=-(1+i+j+k) / 2$ and $R=(i-k) / \sqrt{ } 2$ which is known [10] to be isomorphic to the binary octahedral group of order 48 . Let $T^{*}$ denote the subgroup of $O^{*}$ generated by $P, Q$ and $B$ which is known [10] to be isomorphic to the binary tetrahedral group of order 24 . Let $I^{*}$ denote the finite subgroup of $S^{3}$ generated by $U=(\sqrt{\overline{5}}-1) / 4+i / 2$ $+(\sqrt{5}+1) j / 4$ and $V=-i$ which is known [10] to be isomorphic to the binary icosahedral group of order 120. According to (4.1), we can calculate $\eta\left(S^{3} / T^{*}\right), \eta\left(S^{3} / O^{*}\right)$ and $\eta\left(S^{3} / I^{*}\right)$ by a computer.

$$
\eta\left(S^{3} / T^{*}\right)=49 / 36, \eta\left(S^{3} / O^{*}\right)=121 / 72, \eta\left(S^{3} / I^{*}\right)=361 / 180 .
$$

Hence, by Corollary 3.11, $S^{3} / T^{*}, S^{3} / O^{*}$ and $S^{3} / I^{*}$ can not be globally conformally immersed in $\mathbb{R}^{1}$.

Remark 4.3. As in Example 1.7, so long as the Riemannian metric $g$ of $S^{3} / G$ satisfies the condition that $\left(S^{3}, r^{*} g\right)$ admits an orientationreversing isometry where $r: S^{3} \rightarrow S^{3} / G$ is the covering projection, $\eta\left(S^{3} / G\right)$ can be calculated by $(4.1)$ and $S^{3} / T^{*}, S^{3} / O^{*}$ and $S^{3} / I^{*}$ can not be globally conformally immersed in $\mathbb{R}$.

Next, as an example of Theorem 3.12, we consider 15-dimensional lens space $L^{15}=L(137 ; 1,10,100,41,136,127,37,96)$ which is an example given by Millson in [9]. We assume that the Riemannian metric of $L^{15}$ is partially Pontrjagin flat and has the property (1.8). Then we 
can calculate $\eta\left(L^{15}\right)$ by (1.9). On the one hand, according to the result in [8], $L^{15}$ is known to be stably parallelizable. Hence, $p_{i}\left(T L^{15}\right)=0$ for any $i \geqq 1$. Thus we can put $c=1$. As $N_{4}=14175$, we have

$$
2 c N_{4} \eta\left(L^{15}\right)=120 / 137 \bmod \boldsymbol{Z} \text {. }
$$

Therefore, $L^{15}$ can not be globally conformally immersed with codimension 7 in $\boldsymbol{R}^{22}$.

Remark 4.5. As $L^{15}$ is stably parallelizable, $L^{15}$ can be smoothly immersed with codimension one in $\boldsymbol{R}^{16}$. Moreover, if we give $L^{15}$ the standard metric, $L^{15}$ is locally conformally isomorphic to $\boldsymbol{R}^{15}$.

\section{References}

[1] Atiyah, M. F., Patodi, V. K. and Singer, I. M., Spectral asymmetry and Riemannian geometry I, Math. Proc. Cambridge Philos. Soc., 77 (1975), 43-69.

[2] - Spectral asymmetry and Riemannian geometry II, ibid., 78 (1975), 405432.

[3] Atiyah, M. F. and Singer, I. M., The index of elliptic operator III, Ann. of Math., 87 (1968), 546-604.

[4] Cheeger, J. and Simons, J., Differential characters and geometric invariants, preprint.

[5] Chern, S. S. and Simons, J., Characteristic forms and geometric invariants, Ann. of Math., 99 (1974), 48-69.

[6] Donnelly. H., Eta invariant of a fibered manifold, Topology, 15 (1976), 247-252.

[7] —_, Eta invariants for G-spaces, Indiana Univ. Math. J., 27 (1978), 889-918.

[8] Ewing, J., Moolgavkar, S., Smith, L. and Stong, R. E., Stable parallelizability of lens spaces, J. Pure Appl. Algebra, 10 (1977), 177-191.

[9] Millson, J. J., Examples of nonvanishing Chern-Simons invariants, J. Differential Geometry, 10 (1974), 589-600.

[10] Nakaoka, M., The fixed point theorem and its related topics, Iwanami, 1977, (in Japanese).

[11] Simons, J., Characters associated to a connection, preprint. 